\title{
Micro Computed Tomographic X-ray Imaging (Micro CT): A Versatile and Non- Destructive Method for Biological Specimens.
}

\author{
James C. Williams, Jr. ${ }^{1}$ \\ 1. Department of Anatomy and Cell Biology, Indiana University School of Medicine, Indianapolis, IN, \\ USA.
}

Micro CT utilizes multiple x-ray images of a specimen to reconstruct the three-dimensional structure as visible by differences in $\mathrm{x}$-ray attenuation. As such, it provides a useful tool for many biological specimens. The method is non-destructive, and thus opens the possibility of a specimen being imaged using micro CT and then further analyzed using other methods.

The essence of micro CT is similar to computed tomographic imaging used clinically, but with several significant differences. While in clinical CT the x-ray source and detector are spun around the patient, in micro CT the specimen is rotated within the stationary $\mathrm{x}$-ray beam. This rotation is done in discrete steps, making it more like the old 'step-and-shoot' CT systems than the newer helical CT technologies. The size of the specimen is limited to a few $\mathrm{cm}$ in micro $\mathrm{CT}$, and to obtain the high resolution characteristic of micro CT, the intensities of x-rays are high and the exposure times long, making this kind of micro CT inappropriate for living things (although some intermediate-resolution forms of micro CT are designed for scanning laboratory animals). For the images shown below, scanning time was typical for our specimens, which require scans from 10-60 minutes in length, typically for the collection of $450 \mathrm{x}$ ray images, with the specimen rotated $0.4^{\circ}$ between each image.

The 'tomographic' part of micro CT involves taking the series of $\mathrm{x}$-ray images of the specimen and calculating what arrangement of x-ray-absorbing materials would yield such a series. The most commonly used method for making this calculation is that developed by Feldkamp. [1] Improvements in computing power mean that this calculation — or 'reconstruction' — can be done during the time the next specimen is being scanned. Our Skyscan 1172 system utilizes the graphics processor in the PC to reduce the time of this reconstruction to typically on a few minutes.

We have pioneered the use of micro CT in the study of urinary stones. Micro CT is able to reveal structure within stones to a resolution of just a few micrometers, and a number of mineral types can be accurately distinguished from one another with high spatial resolution. [2] In addition, micro CT allows the screening of large numbers of fragments from a patient so that fragments of distinctive composition can be identified much more easily than by visual inspection. Identification of distinctive regions of a single stone specimen can be beneficial for dissection purposes. For either a group of fragments or for dissection of a single stone, micro CT thus can provide guidance for collecting specimens for further analysis by other means such as Fourier-transform infrared spectroscopy (FT-IR). Finally, we have also shown that micro CT can provide structural/compositional information which can be used to understand the etiologies of stone formation. [3]

The use of micro CT for imaging soft-tissue specimens stained with heavy metals is presently being refined in several laboratories [4] Given that many traditional en bloc histologic stains contain metals in them, these also may be useful to enhance contrast for micro CT imaging of tissues specimens. In this way, methods of the past can be used to provide three-dimensional structural information in 
specimens of tissue. The plan is to highlight some examples of this application of older technology to micro CT in the talk.

\section{References:}

[1] Feldkamp, L.A., L.C. Davis, and J.W. Kress, J Opt Soc Am A 1 (1984), p. 612-619.

[2] Zarse, C.A., J.A. McAteer, A.J. Sommer, S.C. Kim, E.K. Hatt, J.E. Lingeman, A.P. Evan and J.C. Williams. BMC Urology 4 (2004), p. 15.

[3] Williams, J.C., J.E. Lingeman, F.L. Coe, E.M. Worcester and A.P. Evan. Urolithiasis 43 (2015), p. $13-17$.

[4] Khonsari, R.H., C. Healy, A. Ohazama, P.T. Sharpe, H. Dutel, C. Charles, L. Viriot and P. Tafforeau. The Anatomical Record 297 (2014), p. 1803-1807.
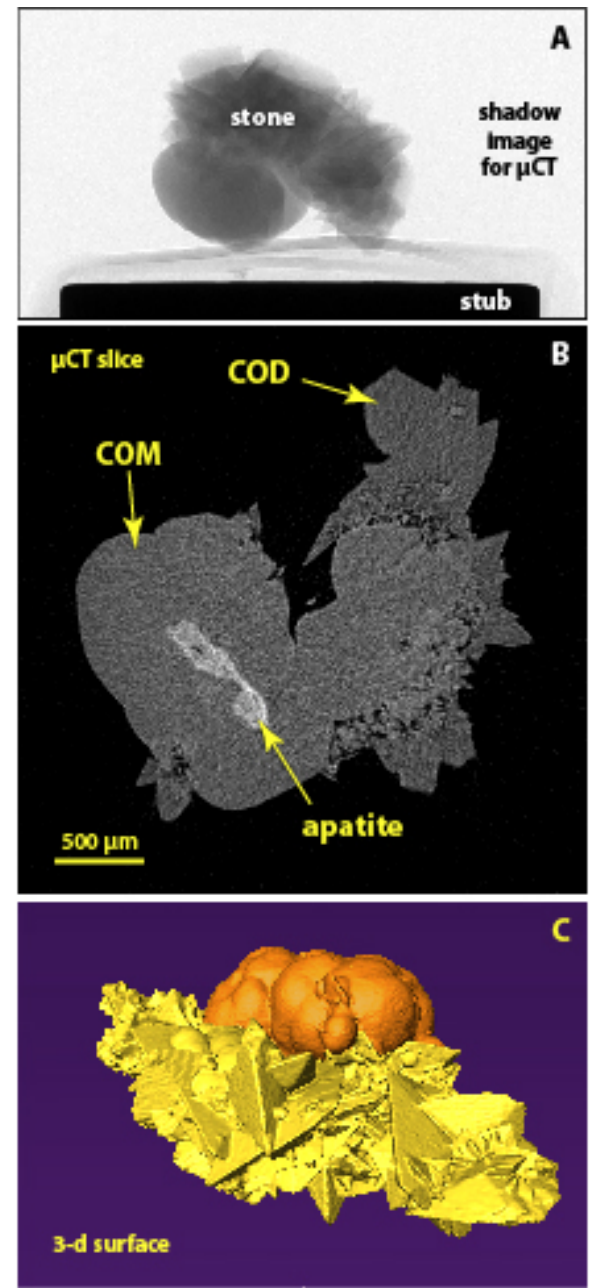

Figure 1. Micro CT imaging of a urinary stone. A. X-ray image taken in the machine. Over 400 such images were collected as the specimen was rotated $0.4^{\circ}$ between each. B. Tomographic reconstruction of a slice through the stone, showing three different mineral types: Calcium oxalate monohydrate (COM), calcium oxalate dihydrate (COD), and calcium phosphate in the mineral form of apatite can clearly be distinguished by $\mathrm{x}$-ray attenuation values (grayscale in the image). C. Surface rendering of the reconstruction, colored to show COD (yellow) and COM (orange). 